\title{
Warum Experten sich für Alleswisser halten
}

\author{
Es ist ein bekanntes psychologisches Phänomen: Fachleute aller Couleur meinen mitunter Fakten \\ zu kennen, die es gar nicht gibt. In raffinierten Studien wird dieses Scheinwissen entlarvt.
}

\begin{abstract}
_ Als Versuchspersonen dienten etwa 600 Männer und Frauen im mittleren Alter von 33 Jahren. In Studie 1 wurden ihnen 15 Begriffe aus der Finanzwelt präsentiert, darunter drei frei erfundene. $92 \%$ glaubten, mindestens einen der Fantasiebegriffe zu kennen. Gleichzeitig sollten die Probanden entweder ihr Wissen selbst einschätzen oder es durch ein Finanz-Quiz prüfen lassen. Ergebnis: Gerade wer sich selbst besser einschätzte oder gute Kenntnisse bewies, „kannte" auch die falschen Begriffe.

In Studie 2 wurde die gleiche Methode auf die Fachgebiete Biologie, Philosophie, Literatur, Mathematik, Architektur, Computerkenntnisse und Kunst des 20. Jahrhunderts ausgedehnt. Dabei zeigte sich, dass die Überschätzung nur die Disziplin betrifft, in der man sich auskennt, nicht aber andere.

In Studie 3 wurden die Versuchspersonen gewarnt, dass einige Begriffe nicht
\end{abstract}

existieren. Trotzdem blieb die Korrelation zwischen Selbsteinschätzung und Selbstüberschätzung erhalten. Für die Autoren zeigt das, dass die Selbstüberschätzung nicht leichtfertig vorgespielt, sondern ehrlich empfunden ist.

In Studie 4 wurde die Selbstüberschätzung kurzfristig manipuliert. Die Probanden mussten zunächst entweder ein leichtes oder ein schweres GeografieQuiz lösen. Erwartungsgemäß führte das zu vielen richtigen bzw. falschen Antworten - und bei den Probanden offenbar zu Stärkung bzw. Minderung der Selbsteinschätzung. Denn wenn danach die Kenntnis von 15 teils realen, teils nicht existenten Orten abgefragt wurde, war die Selbstüberschätzung bei den Probanden mit leichten Fragen gestiegen, mit schweren dagegen gesunken.

\footnotetext{
- Atir S, Rosenzweig E, Dunning D. When Knowledge Knows No Bounds: Self-Perceived Expertise Predicts Claims of Impossible Knowledge. Psychol Sci. 2015;26:1295-303
}

\section{KOMMENTAR}

Sokrates glaubte zu wissen, dass er nichts wusste. Heute begegnet man immer häufiger Menschen, die alles sehr genau wissen. Die Studie zeigt eine besondere Facette dieser Einstellung: Je stärker Menschen glauben, auf einem bestimmten Gebiet Meister zu sein, umso mehr reklamieren sie in dieser Disziplin Wissen, das gar nicht existiert - „impossible knowledge". Das Problem ist also nicht Ignoranz, sondern eine Illusion. Selbstüberschätzung kann Nebenwirkungen haben. So kann ein börseninteressierter Freund seine Expertise überschätzen und fragwürdige Finanzaktionen empfehlen. Auch verliert man die Motivation, sich weiterzubilden, wenn man sich bereits für fast allwissend hält. Im medizinischen Bereich gibt es Ärzte, die Patienten betreuen, für die ein Kollege kompetenter wäre. Und nicht zuletzt gibt es Patienten, die alles besser wissen als der Arzt.

Prof. Dr. med. H. Holzgreve

\section{Auch Radfahren ist nicht nur gesund}

Ein 42-jähriger Mann stellte sich nach einer längeren Radtour mit juckenden Hautknoten im Bereich des Damms vor. An beiden Seiten des Damms sah man erhabene Hautveränderungen von warzenartigem Aussehen. Bioptisch ergaben sich entzündliche dermale Veränderungen, die mit einer nodulären Prurigo vereinbar waren. Dabei handelt es sich um Hautveränderungen mit juckenden, derben Knoten ungeklärter Genese. In der Radfahrergemeinde sind wunde Stellen im Dammbereich durch den Sattel nur zu vertraut und werden mit allerlei blumigen Namen belegt. Im BundeswehrJargon ist der "gelaufene Wolf" notorisch. Ärzte können mit den Veränderungen meistens weniger anfangen, und in Abteilungen für Geschlechtskrankheiten hat das Problem schon gar nichts zu suchen. Wenn es dort auftaucht, führt das gleich zu einer Publikation.

Die Behandlung ist schwierig, vor allem wenn das Radfahren nicht unterbrochen werden kann. Am ehesten helfen noch wattierte Radlerhosen, Deos gegen vermehrtes Schwitzen und sorgfältige Anogenitalhygiene. Auch ein Wechsel der Sattelform sollte erwogen werden. Leider bleibt es nicht bei Hautveränderungen. Bei Männern kann es zu Prostatabeschwerden, Taubheitsgefühlen im Dammbereich und erektiler Dysfunktion kommen.

Es wäre doch schade, wenn die positiven Effekte des Radfahrens durch den falschen Sattel wieder zunichte gemacht würden.

Prof. Dr. med. H. S. FüeßI

- Bateman C, Dilke-Wing G (catherinebateman@btinternet.com). Saddle sores presenting to a genitourinary department. BMJ. 2015;350:h2247

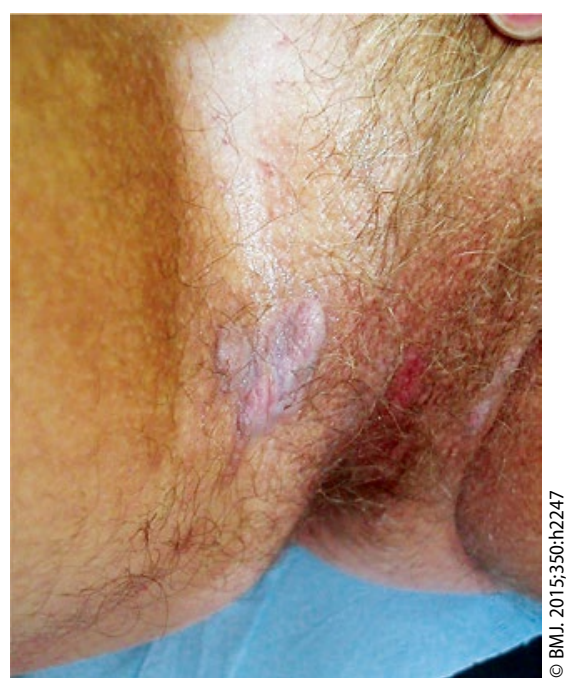

Erhabene, warzenartige, juckende Hautveränderungen beidseitig am Damm. 